\title{
Comparison of High and Medium Iodine Concentrations of Contrast Materials for Preoperative CT Studies in Breast Cancer Patients
}

\author{
Hisashi Yanaihara ${ }^{1}$, Yusuke Inoue ${ }^{2}$, Kazunori Nagahara ${ }^{3}$, Hirofumi Hata ${ }^{3}$, Reiko Woodhams ${ }^{2}$ \\ ${ }^{1}$ Department of Radiology, Kitasato Institute Hospital, Tokyo, Japan \\ ${ }^{2}$ Department of Diagnostic Radiology, Kitasato University School of Medicine, Sagamihara, Japan \\ ${ }^{3}$ Department of Radiology, Kitasato University Hospital, Sagamihara, Japan \\ Email: inoueys34@gmail.com
}

Received August 2, 2012; revised September 3, 2012; accepted September 10, 2012

\begin{abstract}
We compared the contrast effect of high and medium iodine-concentration contrast materials for preoperative CT evaluation of breast cancer patients. Female breast cancer patients who underwent enhanced CT of the chest and upper abdomen before surgery were analyzed retrospectively. High $(370 \mathrm{mg} \mathrm{I} / \mathrm{mL}, 100 \mathrm{~mL})$ or medium $(300 \mathrm{mg} \mathrm{I} / \mathrm{mL}, 125 \mathrm{~mL})$ concentrations of contrast material were injected for $60 \mathrm{sec}$, followed by saline flush, and postcontrast CT images were obtained 90 sec after contrast injection. CT values were assessed for the breast tumor, normal breast parenchyma, liver, aorta, and muscle. For the high and medium concentration agents, 45 and 49 patients were analyzed, respectively. No significant differences between the two contrast agents were found in terms of CT values of the breast tumor, normal breast parenchyma, liver, aorta, or muscle. The tumor-background differences in CT values did not differ significantly between the two agents. A comparable contrast effect was indicated in single-phase CT of breast cancer patients between high and medium iodine-concentration contrast material when the total iodine dose and injection duration were identical.
\end{abstract}

Keywords: CT; Contrast Material; Concentration; Breast Cancer

\section{Introduction}

In computed tomography (CT), contrast enhancement caused by intravenous injection of iodine contrast material depends mainly on the body size of the patient, total iodine dose, and injection rate [1,2]. The iodine concentration of contrast material is another determinant of the contrast effect. Although a higher concentration of contrast material provides higher-quality CT angiograms at a fixed injection rate, in dynamic CT of the abdomen, better enhancement in the aorta and liver has been reported with use of a medium concentration agent than with a high concentration agent when the total iodine dose and injection duration are identical [3-5].

The usefulness of dynamic contrast-enhanced magnetic resonance (MR) imaging for the evaluation of breast cancer is well established [6,7], and multi-phase and single-phase CT has been also applied for this purpose [813]. Although radiation exposure can be a significant problem, CT allows acquisition of images with the patient retaining a posture similar to that during surgery, and the usefulness of CT for the evaluation of the extent of breast cancer has been demonstrated [10-13]. How- ever, investigations of technical factors related to breast CT are limited, and the relationship between contrast effect of breast cancer and iodine concentration of contrast material has not been reported.

We perform enhanced CT of the chest and upper abdomen for preoperative evaluation of breast cancer patients as our routine clinical practice. Because the primary purpose of this CT examination is evaluation of metastatic spread and avoidance of excessive radiation exposure is of critical importance, postcontrast images are obtained at a single time point. Evaluation of the extent of breast cancer is a secondary role of the CT examination, and target images of the breast using a small field-of-view (FOV) are reconstructed from the postcontrast images. The contrast material used for this CT examination was changed from a high iodine concentration agent to a medium iodine concentration agent with no alteration of the total iodine dose or injection duration, based on the previous reports of the superiority of a medium concentration agent. In the present study, we retrospectively compared the contrast effect of the two contrast injection protocols. 


\section{Materials and Methods}

\subsection{Subjects}

Female breast cancer patients who underwent enhanced CT of the chest and upper abdomen for preoperative evaluation from May 6, 2011 to March 31, 2012 were analyzed retrospectively. Inclusion criteria were as follows: 1) MR mammography demonstrated a breast mass of $10 \mathrm{~mm}$ or longer in the long-axis diameter and 2) breast cancer was proven histologically. Patients who received chemotherapy before CT were excluded. The institutional review board approved the current retrospective study and the need for informed consent was waived.

\subsection{Imaging Procedures}

CT was performed using a 64-slice multidetector row CT system (LightSpeed VCT; GE Healthcare, Milwaukee, WI) or 16-slice multidetector row CT system (LightSpeed Ultra16; GE Healthcare). Pre-contrast CT images that covered the liver were obtained. Then, the contrast material was injected for $60 \mathrm{sec}$, followed by a saline flush $(40 \mathrm{~mL})$ at the same rate as the contrast injection, and postcontrast CT images of the chest and upper abdomen were acquired beginning $90 \mathrm{sec}$ after commencement of contrast injection. The contrast material used was a high concentration agent (iopamidol $370 \mathrm{mg} \mathrm{I} / \mathrm{mL}$, 100 mL; Bayer Yakuhin, Ltd., Osaka, Japan) until October 13, 2011 and, thereafter, a medium concentration agent (iohexol $300 \mathrm{mg} \mathrm{I} / \mathrm{mL}, 125 \mathrm{~mL}$; Daiichi-Sankyo, Co., Ltd., Tokyo, Japan). The amount of iodine administered was approximately identical in the two groups and was not adjusted for body size.

For the 64-slice CT system, acquisition parameters were as follows: rotation time, $0.5 \mathrm{sec}$; detector row width, 40 mm; beam pitch, 0.984; FOV, 35 - 40 cm; matrix $512 \times 512$; tube voltage, $120 \mathrm{kV}$; tube current, about $200 \mathrm{~mA}$; section thickness and image interval, $2.5 \mathrm{~mm}$; acquisition time for postcontrast images, 6 sec. The following acquisition parameters were used for the 16-slice system: rotation time, $0.6 \mathrm{sec}$; detector row width, 20 mm; beam pitch, 1.375; FOV, 35 - 40 cm; matrix $512 \times$ 512; tube voltage, $120 \mathrm{kV}$; tube current, about $300 \mathrm{~mA}$; section thickness and image interval, $2.5 \mathrm{~mm}$; acquisition time for postcontrast images, 9 sec. Although we reconstructed target images of the breast using a small FOV, images presenting the entire axial section of the body were used for analysis in the present study.

\subsection{Data Analysis}

Regions of interest (ROIs) were placed in the breast tumor, normal breast parenchyma, liver, aorta, and muscle, and the mean CT value in each ROI was calculated.

For assessment of breast tumor density, an elliptical
ROI was set on the slice presenting the maximal diameter of the tumor (Figure 1). The size of the ROI was determined to be as large as possible, while avoiding inclusion of non-tumorous tissues. In patients with multiple lesions, the largest tumor was analyzed. ROIs for normal breast parenchyma were set ipsilaterally to the tumor. On each of three consecutive slices, a circular ROI of approximately $30 \mathrm{~mm}^{2}$ was drawn, minimizing inclusion of fat tissues. The average value was calculated from the three mean CT values of the three ROIs and was regarded as representative of the density of normal breast parenchyma. The tumor-background difference in CT value was computed as the postcontrast CT value of the breast tumor minus that of normal breast parenchyma.

For the liver, a circular ROI of approximately 100 $\mathrm{mm}^{2}$ was placed in each of the anterior segment of the right hepatic lobe, posterior segment of the right lobe, and medial segment of the left lobe, avoiding inclusion of vascular structures, on the slice presenting the right

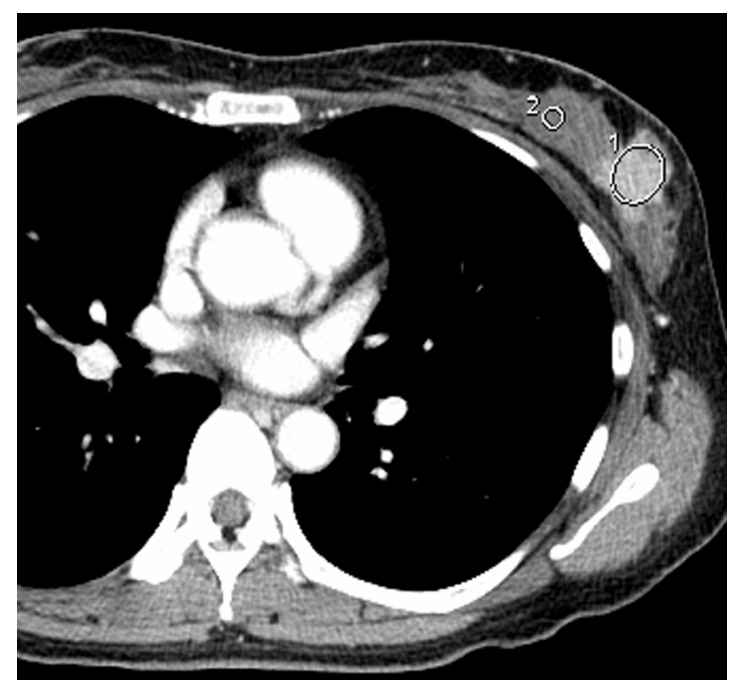

Figure 1. ROIs for the breast tumor and normal breast parenchyma.

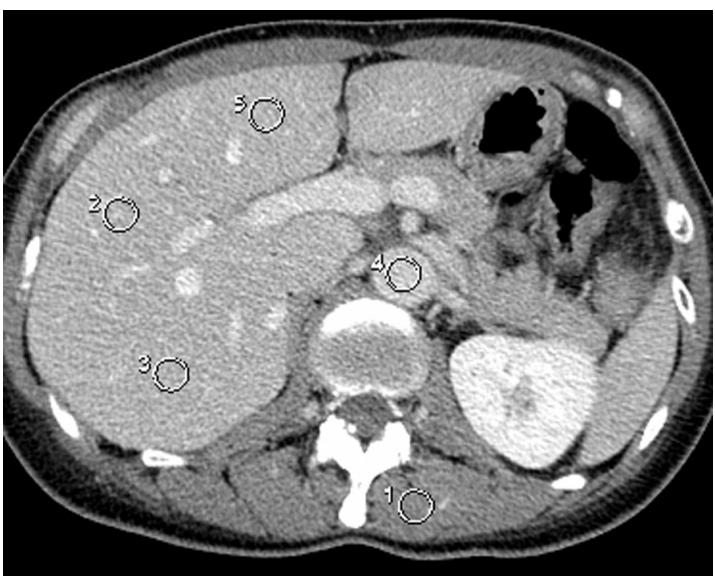

Figure 2. ROIs for the liver, aorta, and muscle. 
main branch of the portal vein (Figure 2). The CT value of the liver was defined as the average of the mean CT values of the three ROIs. For the aorta and muscle, a circular ROI of approximately $100 \mathrm{~mm}^{2}$ was placed in the abdominal aorta and erector muscle of spine, respectively, on the slice used for assessment of liver density. Attention was paid to minimize inclusion of fat in the muscle ROI. ROIs for the liver, aorta, and muscle were set at the same position on the precontrast and postcontrast images, and the postcontrast increase in CT value was calculated.

\subsection{Statistical Analysis}

Values were compared using an unpaired Student's $t$-test between high and medium concentrations of contrast material. A p value less than 0.05 was deemed to indicate statistical significance.

\section{Results}

For the high and medium concentrations of contrast materials, 45 and 49 patients were analyzed, respectively. No significant differences in age, body weight, and diameter of tumor analyzed were found between the two groups (Table 1).

No significant differences between high and medium concentration agents were found in terms of the CT values of the breast tumor, normal breast parenchyma, liver, aorta, or muscle (Table 2). The tumor-background difference did not differ significantly between the two agents. There were no significant differences between the two agents in terms of the postcontrast increase in CT values in the liver, aorta, and muscle.

\section{Discussion}

In the present retrospective study, we compared the contrast effect of high and medium iodine concentrations of contrast materials in CT examinations for preoperative evaluation of breast cancer patients. Dynamic MR imaging is the primary imaging method for evaluation of the characteristics and extent of a breast mass at our hospital. CT is used mainly for assessment of metastatic spread, and multi-phase postcontrast imaging is not performed to

Table 1. Patient characteristics.

\begin{tabular}{lccc}
\hline & \multicolumn{2}{c}{ Concentration } & \multirow{2}{*}{ p value } \\
\cline { 2 - 3 } & High & Medium & \\
\hline Number & 45 & 49 & \\
Age (years) & $57.2 \pm 11.0$ & $60.8 \pm 12.8$ & 0.149 \\
Body weight (kg) & $52.9 \pm 6.3$ & $53.7 \pm 11.0$ & 0.368 \\
Tumor size (mm) & $21.2 \pm 8.7$ & $21.5 \pm 10.5$ & 0.609 \\
\hline
\end{tabular}

Values are expressed as the mean \pm standard deviation. Tumor size $=$ long-axis diameter measured on MR mammography.
Table 2. Results of CT values.

\begin{tabular}{lccc}
\hline & \multicolumn{2}{c}{ Concentration } & p value \\
\cline { 2 - 4 } & High & Medium & \\
\hline Postcontrast CT value & & & \\
Breast tumor & $87.6 \pm 28.3$ & $89.5 \pm 25.9$ & 0.734 \\
Normal breast parenchyma & $17.7 \pm 22.2$ & $16.1 \pm 14.9$ & 0.684 \\
Liver & $135.7 \pm 21.8$ & $133.1 \pm 16.4$ & 0.507 \\
Aorta & $182.2 \pm 16.4$ & $182.4 \pm 16.7$ & 0.952 \\
Muscle & $65.6 \pm 13.9$ & $64.4 \pm 23.9$ & 0.760 \\
Tumor-background & $69.9 \pm 32.9$ & $73.4 \pm 27.3$ & 0.576 \\
difference & & & \\
Postcontrast increase & $76.8 \pm 15.1$ & $74.1 \pm 15.0$ & 0.398 \\
Liver & $141.6 \pm 15.7$ & $140.6 \pm 16.0$ & 0.752 \\
Aorta & $21.0 \pm 11.8$ & $23.0 \pm 6.9$ & 0.323 \\
Muscle &
\end{tabular}

Values are expressed as the mean \pm standard deviation.

reduce radiation exposure. Precontrast images are acquired for assessment of liver lesions, and the breast is imaged only at a single time point after contrast injection. Thus, estimation of the postcontrast increase in CT value was not feasible for the breast, and postcontrast CT values of breast cancer and normal breast parenchyma were compared between the high and medium concentrations of contrast material. On the postcontrast CT images, neither the CT value of the breast cancer nor tumor-background difference differed significantly between the two contrast agents, suggesting a comparable contrast effect for breast cancer. Additionally, contrast enhancement in the liver and abdominal aorta was similar between the two contrast agents. No differences in the detectability of metastatic liver lesions or visualization of vascular structures were suggested. These results indicate equivalence of the high and medium concentration agents with an identical total iodine dose and identical injection duration.

In contrast to our results, previous studies have demonstrated a larger increase in CT value after injection of a medium concentration agent than after injection of a high concentration agent at an identical total iodine dose and identical injection duration [3-5]. This effect was more evident at an earlier phase after contrast injection. In the present study, postcontrast images were acquired $90 \mathrm{sec}$ after the start of contrast injection of 60-sec duration. The optimal delay time to depict breast cancer has been reported to be 80 sec after contrast injection of 33-sec duration [14]. Although the delay time of our protocol appears to be acceptable for evaluation of the extent of breast cancer-the secondary purpose of the CT examination-dynamic breast CT may be used for more comprehensive assessment of breast tumors, including differentiation of malignant from benign lesions [8-10]. The contrast effect at an earlier phase remains to be compared 
between the two contrast protocols.

In our contrast method, injection of contrast material is followed by a saline flush to ensure efficient use of the contrast material $[15,16]$. Without a saline flush, a fraction of contrast material is retained in the injection route and the venous system from the upper extremity to the superior vena cava, and this fraction does not contribute to intended contrast enhancement. The volume of retained agent is assumed to be similar irrespective of the concentrations of contrast material. Therefore, the amount of iodine retained would be larger for a higher concentration agent, and the amount of iodine that contributes to effective contrast enhancement would be larger for a lower concentration agent. A saline flush is not always performed for contrast injection in CT. The present study indicated a comparable contrast effect for high and medium concentrations of contrast materials with the use of a saline flush, which may suggest the superiority of a medium concentration agent.

\section{Conclusion}

A comparable contrast effect was indicated in singlephase CT for preoperative evaluation of breat cancer patients between high and medium iodine concentrations of contrast material when the total iodine dose and injection duration were identical and a saline flush was performed. The contrast effect at an earlier phase after injection and without a saline flush remains to be investigated.

\section{Acknowledgements}

The corresponding author has received research grants from Bayer Yakuhin, Ltd. and Daiichi-Sankyo, Co., Ltd.

\section{REFERENCES}

[1] Y. C. Cheung, S. C. Chen, S. H. Ueng, S. F. Ko and Y. L. Wan, "Dynamic Enhanced Computed Tomography Values of Locally Advanced Breast Cancers Predicting Axilla Nodal Metastasis after Neoadjuvant Chemotherapy,” Journal of Computer Assisted Tomography, Vol. 33, No. 3, 2009, pp. 422-425. doi:10.1097/RCT.0b013e31818521b3

[2] H. Kondo, M. Kanematsu, S. Goshima, Y. Tomita, M. J. Kim, N. Moriyama, et al., "Body Size Indexes for Optimizing Iodine Dose for Aortic and Hepatic Enhancement at Multidetector CT: Comparison of Total Body Weight, Lean Body Weight, and Blood Volume,” Radiology, Vol. 254, No. 1, 2010, pp. 163-169. doi:10.1148/radiol.09090369

[3] K. Awai, M. Inoue, Y. Yagyu, M. Watanabe, T. Sano, S. Nin, et al., "Moderate versus High Concentration of Contrast Material for Aortic and Hepatic Enhancement and Tumor-to-Liver Contrast at Multi-Detector Row CT," Radiology, Vol. 233, No. 3, 2004, pp. 682-688. $\underline{\text { doi:10.1148/radiol.2333031617 }}$
[4] T. Ichikawa, S. M. Erturk and T. Araki, "Multiphasic Contrast-Enhanced Multidetector-Row CT of Liver: Contrast-Enhancement Theory and Practical Scan Protocol with a Combination of Fixed Injection Duration and Patients' Body-Weight-Tailored Dose of Contrast Material," European Journal of Radiology, Vol. 58, No. 2, 2006, pp. 165-176. doi:10.1016/j.ejrad.2005.11.037

[5] J. K. Han, A. Y. Kim, K. Y. Lee, J. B. Seo, T. K. Kim, B. I. Choi, et al., "Factors Influencing Vascular and Hepatic Enhancement at CT: Experimental Study on Injection Protocol Using a Canine Model," Journal of Computer Assisted Tomography, Vol. 24, No. 3, 2000, pp. 400-406. doi:10.1097/00004728-200005000-00008

[6] L. R. Medeiros, C. S. Duarte, D. D. Rosa, M. I. Edelweiss, M. Edelweiss, F. R. Silva, et al., "Accuracy of Magnetic Resonance in Suspicious Breast Lesions: A Systematic Quantitative Review and Meta-Analysis,” Breast Cancer Research and Treatment, Vol. 126, No. 2, 2011, pp. 273285. doi:10.1007/s10549-010-1326-9

[7] C. K. Kuhl, “Current Status of Breast MR Imaging. Part 2. Clinical Applications,” Radiology, Vol. 244, No. 3, 2007, pp. 672-691. doi:10.1148/radiol.2443051661

[8] A. Perrone, L. L. Mele, S. Sassi, M. Marini, L. Testaverde, L. Izzo, et al., "MDCT of the Breast," American Journal of Roentgenology, Vol. 190, No. 6, 2008, pp. 1644-1651. doi:10.2214/AJR.07.3145

[9] K. Miyake, K. Hayakawa, M. Nishino, Y. Nakamura, T. Morimoto, Y. Urata, et al., "Benign or Malignant? Differentiating Breast Lesions with Computed Tomography Attenuation Values on Dynamic Computed Tomography Mammography," Journal of Computer Assisted Tomography, Vol. 29, No. 6, 2005, pp. 772-779. doi:10.1097/01.rct.0000178712.32547.53

[10] M. Inoue, T. Sano, R. Watai, R. Ashikaga, K. Ueda, M. Watatani, et al., "Dynamic Multidetector CT of Breast Tumors: Diagnostic Features and Comparison with Conventional Techniques," American Journal of Roentgenology, Vol. 181, No. 3, 2003, pp. 679-686.

[11] M. Tozaki, T. Kobayashi, S. Uno, K. Aiba, H. Takeyama, H. Shioya, et al., "Breast-Conserving Surgery after Chemotherapy: Value of MDCT for Determining Tumor Distribution and Shrinkage Pattern," American Journal of Roentgenology, Vol. 186, No. 2, 2006, pp. 431-439. doi:10.2214/AJR.04.1520

[12] H. Nakahara, K. Namba, H. Wakamatsu, R. Watanabe, H. Furusawa, M. Shirouzu, et al., "Extension of Breast Cancer: Comparison of CT and MRI," Radiation Medicine, Vol. 20, No. 1, 2002, pp. 17-23.

[13] T. Uematsu, M. Sano, K. Homma, H. Makino, M. Shiina, S. Kobayashi, et al., "Staging of Palpable T1-2 Invasive Breast Cancer with Helical CT,” Breast Cancer, Vol. 8, No. 2, 2001, pp. 125-130. doi:10.1007/BF02967491

[14] S. Kuroki-Suzuki, Y. Kuroki, T. Ishikawa, H. Takeo and N. Moriyama, "Diagnosis of Breast Cancer with Multidetector Computed Tomography: Analysis of Optimal Delay Time after Contrast Media Injection,” Clinical Imaging, Vol. 34, No. 1, 2010, pp. 14-19. doi:10.1016/j.clinimag.2009.03.004

[15] P. Haage, T. Schmitz-Rode, D. Hübner, W. Piroth and R. 
W. Günther, "Reduction of Contrast Material Dose and Artifacts by a Saline Flush Using a Double Power Injector in Helical CT of the Thorax," American Journal of Roentgenology, Vol. 174, No. 4, 2000, pp. 1049-1053.

[16] K. D. Hopper, T. J. Mosher, C. J. Kasales, T. R. TenHave,
D. A. Tully and J. S. Weaver, “Thoracic Spiral CT: Delivery of Contrast Material Pushed with Injectable Saline Solution in a Power Injector,” Radiology, Vol. 205, No. 1, 1997, pp. 269-271. 